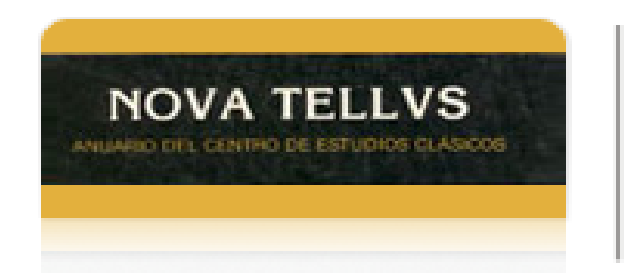

Nova Tellus

ISSN: 0185-3058

novatelu@servidor.unam.mx

Centro de Estudios Clásicos

México

Valdés García, Hilda Julieta

Villa di Mecenate: una disertación arqueológica de Pedro José Márquez

Nova Tellus, vol. 28, núm. 1, 2010, pp. 303-337

Centro de Estudios Clásicos

Distrito Federal, México

Disponible en: http://www.redalyc.org/articulo.oa?id=59115484010

- Cómo citar el artículo

- Número completo

- Más información del artículo

- Página de la revista en redalyc.org

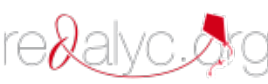

Sistema de Información Científica

Red de Revistas Científicas de América Latina, el Caribe, España y Portugal

Proyecto académico sin fines de lucro, desarrollado bajo la iniciativa de acceso abierto 


\title{
Villa di Mecenate: una disertación arqueológica de Pedro José Márquez
}

\author{
Hilda Julieta VALDÉS GARCÍA \\ Universidad Nacional Autónoma de México \\ helade01@hotmail.com
}

\begin{abstract}
RESUMEN: Pedro José Márquez Durán radicó en Roma junto con sus hermanos de orden a causa del mandato de expulsión de Carlos III. Allí se dedicó al estudio de la teoría arquitectónica y estética. A finales del siglo XVIII, el embajador español en Italia, José Nicolás de Azara, llevó a cabo una expedición en Tívoli, en el emplazamiento de las ruinas conocidas como la Villa di Mecenate, en la que participaron los dos pensionados de la Real Academia de Bellas Artes de San Fernando de Madrid, Silvestre Pérez y Evaristo del Castillo, y el jesuita mexicano Pedro José Márquez. Este último elaboró una disertación para defensa de la denominación del lugar.
\end{abstract}

\section{Villa di Mecenate: An archeologycal dissertation by Pedro José Márquez}

ABSTRACT: Pedro José Márquez Durán settled in Rome with his Jesuit brothers because of the expulsion ordered by Carlos III. There, he dedicated to the study of architectonic and esthetics theory. To the end of the 18th century, the Spanish ambassador in Italy, José Nicolás de Azara, made an expedition to Tivoli at the ruins of the well known Villa di Mecenate. Silvestre Pérez and Evaristo del Castillo, pensioners of the Royal Academy of Fine Arts of San Fernando in Madrid, took part of it and the mexican Jesuit Pedro José Márquez wrote a dissertation to defend the name of the place.

PALABRAS ClAVE: ruinas romanas, Tívoli, Villa di Mecenate, Santuario d'Ercole Vincitore, Pedro José Márquez.

KEYWORDS: Roman archeological ruins, Tivoli, Villa di Mecenate, Ercole Vincitore Sanctuary, Pedro José Márquez.

RECEPCIÓN: 1 de abril de 2010.

ACEPTACIÓN: 26 de mayo 2010. 


\title{
Villa di Mecenate: una disertación arqueológica de Pedro José Márquez ${ }^{1}$
}

\author{
Hilda Julieta VALDÉs GARCíA
}

Pedro José Márquez Durán (1741-1820), originario de San Francisco del Rincón, Guanajuato, ingresó a los veinte años de edad como novicio de la Compañía de Jesús en Tepotzotlán. En 1767, el emperador Carlos III emitió la Pragmática Sanción que ordenaba la expulsión de los jesuitas de todos los reinos españoles; ésta sorprendió al joven Márquez siendo estudiante de teología en el Colegio de San Pedro y San Pablo. Desde el puerto de Veracruz, junto con sus hermanos de orden, marchó hacia el destierro. En Bolonia fue ordenado sacerdote en 1769; sin embargo, será en Roma donde pasará la mayor parte de los años del exilio y verán la luz sus publicaciones sobre astronomía, teoría estética, arquitectura y arqueología mesoamericana y clásica. ${ }^{2}$

El padre Márquez formó parte del grupo de jesuitas expulsos mexicanos entre los que sobresalieron Diego José Abad, Francisco Javier Alegre, Francisco Javier Clavijero, Andrés Cavo y Rafael Landívar, por mencionar algunos. Alejados del ocio, se dedicaron al estudio y lograron publicar obras sobre diversas áreas del conocimiento como estética, filosofía, historia y poesía.

\footnotetext{
${ }^{1}$ El presente estudio fue realizado dentro de la línea de investigación de la tesis doctoral "Vitruvio en la obra arqueológica de Pedro José Márquez, un estudio de fuentes" que actualmente desarrollo en el Posgrado en Letras Clásicas de la Facultad de Filosofía y Letras, UNAM, con el apoyo del Consejo Nacional de Ciencia y Tecnología.

${ }^{2}$ Cf. Valdés, 2008, pp. 255-256.
}

$305 \quad$ NOVA TELLVS, $28 \cdot 1,2010$ 
En la Ciudad eterna, Márquez entró en comunicación con el diplomático español José Nicolás de Azara (1730-1804), "reconocido hombre de letras, corresponsal infatigable, memorialista secreto, anticuario aficionado, apasionado coleccionista y bibliófilo", 3 quien se relacionó y mantuvo correspondencia con un nutrido grupo de intelectuales y artistas importantes de su tiempo: Winckelmann, Mengs, Milizia, G. Hamilton, Volpate, Canova y los abates Fea y Visconti. ${ }^{4}$ Como embajador de España en Italia, demostró ávido interés por la cultura y supo aprovechar su posición y poder social para fungir como importante mecenas:

Los jesuitas expulsos en Italia están bajo su autoridad y uno de ellos -Esteban de Arteaga - fue su secretario. También dependen de él los pensionados de la Academia de San Fernando en Roma (están bajo su órbita anticuaria los arquitectos Silvestre Pérez, Evaristo del Castillo e Isidoro González Velázquez, a los que relaciona con Francesco Milizia y el mejicano Pedro José Márquez).$^{5}$

Se sabe que Azara fue partidario de la extinción de la Compañía; sin embargo, una vez extinta, nada impidió que diera protección a algunos de sus miembros, pues, ante todo, su espíritu humanista lo llevó a reconocer el intelecto de estos varones.

El diplomático Azara fue considerado uno de los "más sabios anticuarios, opinión que justifican sus escelentes escavaciones en Tívoli y en otros puntos"; 6 poseía una casa de campo en esta ciudad cercana a Roma y, como todo hombre

\footnotetext{
${ }^{3}$ Sánchez, 1997, p. 9.

${ }^{4}$ Idem, 1993, pp. 9-10. En este interesantísimo estudio Gabriel Sánchez nos permite apreciar, a través de la vida de este personaje, el ambiente político y cultural de Italia y España en el siglo XVIII.

${ }^{5}$ Ibid.

${ }^{6}$ Sobre el interés anticuario de Azara cf. las obras de Castellanos de Losada, 1850, p. 5; 1856, passim.
} 
ilustrado, se sintió atraído por la "fiebre anticuaria", pasando de la lectura de los textos a las expediciones: el siglo de las luces exigía pruebas, como lo demuestra la vasta producción de estudios comparativos surgidos en ese tiempo. A este personaje se atribuye el descubrimiento de los restos de la Villa de Mecenas entre los años 1793-1794. ${ }^{7}$

\section{Villa di Mecenate, historia de una expedición}

La historia del texto de la Villa di Mecenate nos es transmitida por el mismo Pedro José Márquez. José Nicolás de Azara, al tener conocimiento de que el gobierno realizaba obras sobre el emplazamiento de la denominada Villa de Mecenas, se ocupó de las gestiones necesarias para excavar en las ruinas antes de que

si soprapponessero le opere delle moderne ferriere, fece immantimenti ricavarne i disegni in sette tavole, nella cui delineazione riuscirono con onore i due abili Architetti Spagnuoli D. Silvestro Perez, e D. Evaristo del Castillo pensionati dell' Accademia di Madrid. ${ }^{8}$

Satisfecho Azara con los opúsculos que Márquez había publicado, Delle case di città degli antichi Romani (1795) y Delle ville di Plinio il giovane (1796), instó al jesuita mexicano a que emprendiera la descripción de la Villa:

\footnotetext{
${ }^{7}$ Mora, 1996, p. 72.

${ }^{8}$ Márquez, 1812, p. 4. (En la transcripción de las citas en italiano he respetado la ortografía que aparece en éstas.) Silvestre Pérez radicó en Roma de 1790 a 1796, donde realizó numerosos diseños de monumentos antiguos romanos. De las siete tablas que menciona aquí Márquez, sólo una, la más representativa, está firmada por los dos becarios y corresponde a la Tabla I de las Illustrazioni (fig. 1). Pérez envió a la Academia de San Fernando de Madrid un total de veintisiete diseños sobre el Templo de Júpiter Stator, la Quinta Tibutina de Mecenas y el Teatro Marcelo. Cf. García, 1998, pp. 159-160.
} 
Coi disegni a mia disposizione portatomi più volte a registrare ocularmente gli avanzi della fabbrica ed a confrontare l'uno, coll'altro, mi accinsi a distendere la descrizione di tutto l'edifizio: questa finita, la lesse detto Signore, e mi palesò la sua resoluzione di pubblicare ogni cosa con magnificenza da rico Cavaliere, conoscitore del buono, amatore delle belle Arti, e protettore degli studiosi... ${ }^{9}$

En efecto, el embajador español tenía sumo interés en la publicación de esta obra, así lo expresó en 1800 a su amigo Giambattista Bodoni, reconocido impresor y tipógrafo italiano:

O' un opera curiosa di Architettura sul Dorico e sulle rovine di Mecenate a Tivoli, che feci disegnare in tempi felici magnificamente, ma i disegni sono restati in Roma con tutte le mie altre cose. Al mio ritorno in Italia pensaremo a pubblicare questa opera, con qualche altra coseta di più. ${ }^{10}$

Empero, desafortunadamente, Azara pudo ver únicamente impresa la obra sobre el Dórico, ${ }^{11}$ la cual le había sido enviada por Márquez a París ${ }^{12}$ con una carta en la que le solicitaba le devolviese varios manuscritos, entre éstos, el de la Villa de Mecenas:

Por medio de D. Juan Paris remito a V. Ex. una copia del libro sobre el Dorico, que se acaba de imprimir... Una vez impreso el discurso dicho sobre el dorico, ya el manuscrito, que allá tiene V. Ex. no le sirve; por tanto, si a V. Ex, le parece, en lo habiendo oportunidad, me lo podra remitir junto con los otros dos: estimaria mucho tener aqui el de Mecenas por la planta de la Vila (sic), que lo acompaña; pues, aunque conservé el borrador del

\footnotetext{
${ }^{9}$ Idem, 1812, pp. 4-5.

${ }^{10}$ Carta de Azara a Bodoni, fechada el 4 de marzo de 1800, Barcelona, en Sánchez, 1997, p. 176.

${ }^{11}$ Márquez, 1803.

${ }^{12}$ Azara salió de Roma en 1798, cuando fue trasferido a París, donde murió en 1804.
} 
discurso, no me quedó copia de dicha planta, y esa me serviria grandemente en falta de los dibujos de V. Ex. Mientras no se pueden entresacar de los caxones de sus libros por no saberse en qual estan. ${ }^{13}$

El discurso Villa di Mecenate que presentamos coincide con la descripción anterior y con la advertencia final en las Illustrazioni: "Nello squarcio della detta dissertazione, che si lesse in un' adunanza della nostra Accademia si sono raccolte le ragioni, e le autorità che dimostrano essere stato l' edifizio di cui si tratta la Villa di C. Cilnio Mecenate...". ${ }^{14}$

Así pues, colegimos que el ejemplar de la Villa de Mecenas en poder de nuestro jesuita era el borrador sin ilustración alguna; que el discurso había sido leído en la Academia de Arqueología y, finalmente, que en el texto se desarrollaban los argumentos necesarios para demostrar que la villa era la de Mecenas.

\section{Villa di Mecenate, dissertazione}

Villa di Mecenate viene a incrementar el número de manuscritos recuperados de las disertaciones arqueológicas del padre Márquez. Pocos biógrafos antiguos y modernos del guanajuatense hacen referencia al escrito; empero, sólo aquellos que profundizaron en sus obras ${ }^{15}$ consideran que su extensión es mayor a su versión definitiva, Illustrazioni della Villa di Mecenate in Tivoli, ${ }^{16}$ debido a las siguientes palabras de Márquez: "Ed ecco in ristretto quello che più distesamente abiamo scritto nella sopracitata dissertazione, la quale insieme coi

\footnotetext{
${ }^{13}$ Carta de Pedro José Márquez a Nicolás de Azara, Roma, 18 de julio de 1803. BNM, ms. 20084 ${ }^{12}$.

${ }^{14}$ Márquez, 1812, p. 5.

${ }^{15}$ Couto, 1854, p. 144; Decorme, "Bibliografía del P. Márquez”, p. 3.

${ }^{16}$ Márquez, 1812.
} 
Commentarj sopra la Vita di C. Cilnio Mecenate, aspetteranno altro tempo per veder la pubblica luce..."17

El texto Villa de Mecenate ${ }^{18}$ consta de diez fojas; en la última, aparece el nombre de $P^{r}$. Márquez. De acuerdo con lo expuesto anteriormente, se trata de un borrador del discurso original; sin embargo, la caligrafía del escrito, de excelente factura, no corresponde a la utilizada por Márquez en otros hológrafos conservados, ni a las otras manos que se distinguen en manuscritos más amplios de nuestro autor. A pesar de esta particularidad, no cabe duda de la autoría. La versión definitiva impresa en 1812 contiene, en general, la misma argumentación.

La estructura de la disertación es muy sencilla, casi lineal. Para demostrar que las ruinas de Tívoli descritas corresponden a la Villa de Mecenas, Márquez presenta sólo dos argumentos: la extensión de las ruinas de la construcción concuerda con la opulencia del propietario y el nombre tradicionalmente dado a este complejo arquitectónico por los tiburtinos ab immemorabili reduce cualquier otro argumento contrario.

Para fundamentar su discurso y elevarlo a nivel de la erudición, Márquez lo enriquece no sólo con citas de autores clásicos, sino con referencias de "tutti gli scrittori delle cose tiburtine", autores reconocidos en el ámbito de la ciencia anticuaria como Zappi, Marzio, Volpi, Kircher, Holstein, entre otros, seguían siendo autoridades en la materia.

Y para aquellos que siguiendo a Pirro Ligorio, célebre arquitecto, pintor y anticuario del siglo XVI, atribuyen la propiedad de la Villa al emperador Augusto, Márquez sugiere

\footnotetext{
${ }^{17}$ Idem, p. 15; Gutiérrez, 1994, p. 86, afirma que el manuscrito anónimo Comentari sopra la vita di Mecenate, resguardado en el Fondo Jesuítico de la Biblioteca Nacional "Vittorio Emmanuele", Roma, núm. 113 (2242) es obra de nuestro autor.

${ }^{18}$ Lo hallé al final del ejemplar de las Esercitazioni architettoniche en el acervo del Archivo Histórico de la Provincia Mexicana de la Compañía de Jesús en México, D. F. Cf. Valdés, 2007; Valdés, 2008.
} 
se reconsidere esta postura después de la lectura atenta de la Vida de Augusto de Suetonio. Al Sr. Chaupy, contemporáneo suyo, quien propone que el emplazamiento corresponde a una basílica o foro, lo remite a la obra de Vitruvio, el "maestro", quien en su libro quinto da los preceptos para la construcción de estas obras públicas...

El discurso termina ex abrupto; de manera categórica el autor sostiene que el mejor argumento es la tradición, pues sentencia que "con questo nome, e non con altri chiunque vada a Tivoli può ritrovarse l'edificio di cui si tratta".

Una pequeña nota suelta o testigo postscripto con letra de nuestro autor, colocado entre las fojas, advierte los materiales adicionales que deben ser incorporados al discurso en la versión definitiva: "Questa iscrizione ${ }^{19}$ si riporta nella spiegazione della tavole n. 13 come anche l'altra di cui si parla in appresso. Qui dovra seguire la spiegazione delle tavole, che dimostrano la pianta, e le elevazioni della villa".

Este breve testimonio confirma el carácter de escrito previo del documento. La información sobre el número de ilustraciones o tablas preparadas para acompañar la exposición nos permite tener una idea de la extensión del trabajo final. Por el mismo Márquez sabemos que la obra Illustrazioni della Villa di Mecenate (que únicamente cuenta con cuatro tablas) era sólo una parte del estudio completo que había desarrollado y que aún esperaba publicar. ${ }^{20}$

En esta época de vertiginoso avance científico, resulta difícil valorar con justicia el tipo de documentos como el que presentamos. Es verdaderamente notable la cantidad de escritos sobre antigüedades que se publicaron en el siglo XVIII. La Villa di Mecenate había sido leído en la Academia de Arqueo-

\footnotetext{
${ }^{19}$ Una de estas inscripciones se refiere a la Via Tecta o Porta oscura (fig. 2), señalando la intervención de los cuadrúnviros, quienes cubrieron la Vía en aquel sitio por orden del senado. Cf. Márquez, 1812, pp. 7-8.

${ }^{20}$ Idem, p. 5; 15.
} 
logía en la última década del siglo XVIII. ${ }^{21}$ Es posible conocer la recepción que tuvo esta disertación, pues hemos visto la intención del humanista Azara de publicar la versión definitiva con las ilustraciones que había mandado realizar ex profeso a diseñadores talentosos. El autor de la obra, Pedro José Márquez, fue recibido en 1810 como miembro de la ilustre Academia Arqueológica de Roma. ${ }^{22}$ Dos años más tarde, bajo el auspicio de esta institución, fue publicada, como hemos mencionado, la versión definitiva del discurso bajo el nombre de Illustrazioni della Villa di Mecenate in Tivoli.

En cuanto a las ilustraciones, que merecen un estudio aparte, algunas se incluyeron en las guías turísticas romanas de la época como la del arqueólogo Antonio Nibby y la de Giovanni Battista Cipriani, ${ }^{23}$ grabador de la Tabla I de las Illustrazioni (fig. 1).

El discurso de la Villa di Mecenate es, sin duda, un claro ejemplo de la manera en que la ciencia anticuaria - sin ningún otro recurso, excepto el de las fuentes clásicas - se fue abriendo camino hasta llegar a ser la ciencia arqueológica que es hoy día. El emplazamiento de la Villa de Mecenas descrito por Márquez finalmente fue interpretado como el Santuario de Hércules vencedor; durante mucho tiempo ambos nombres serían utilizados para denominar el lugar.

En 1816 nuestro jesuita regresó a México; cuatro años después moriría en su patria quien debería ser recordado como el primer mexicano en participar activamente en las expediciones arqueológicas europeas.

\footnotetext{
${ }^{21}$ Vid. supra, Villa di Mecenate, historia de una expedición.

${ }^{22}$ Fue miembro de las Academias de Bellas Artes de Madrid (1797), en 1804 ya pertenecía a las Academias de Florencia y Bolonia. En 1809 ingresó a la Academia de San Luca (Roma). Cf. Valdés, 2008, p. 256; Fernández, 1988, p. 447.

${ }^{23}$ Nibby, 1819. Es posible que este autor se haya basado en las Illustrazioni di Márquez para realizar su descripción de la Villa de Mecenas; Cipriani, 1835.
} 


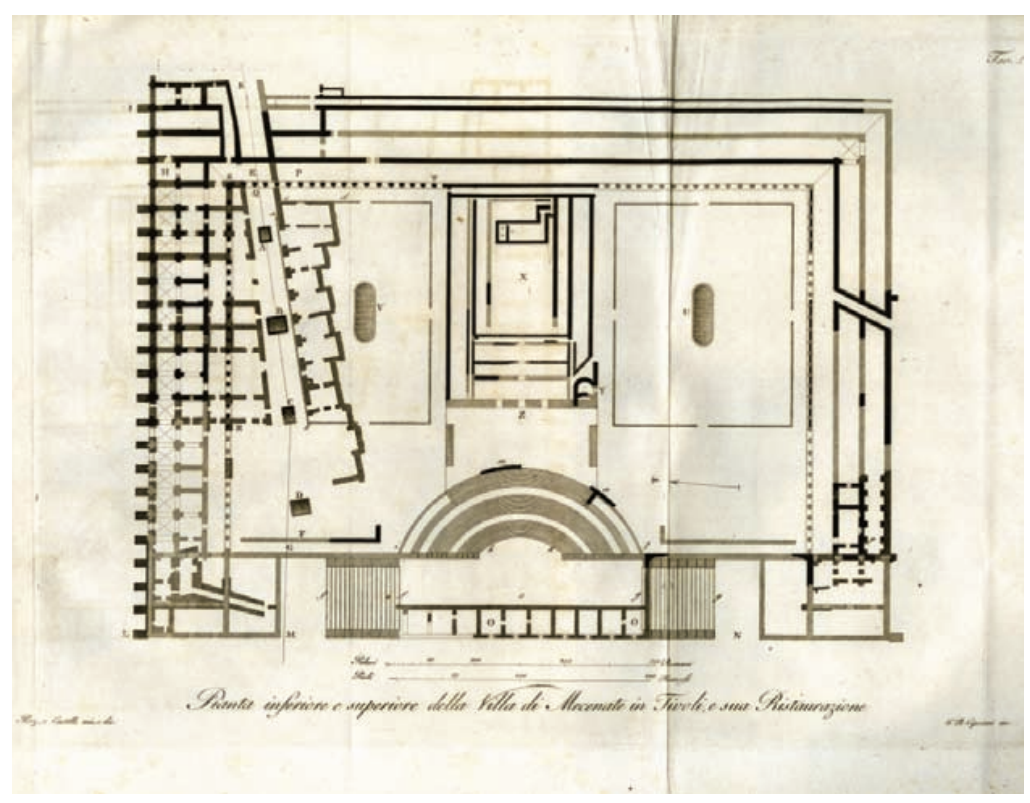

FIG. 1. Pianta inferiore e superiore della Villa di Mecenate in Tivoli, e sua Ristaurazione ${ }^{24}$

\footnotetext{
${ }^{24}$ Márquez, 1812, Tav. I.
} 


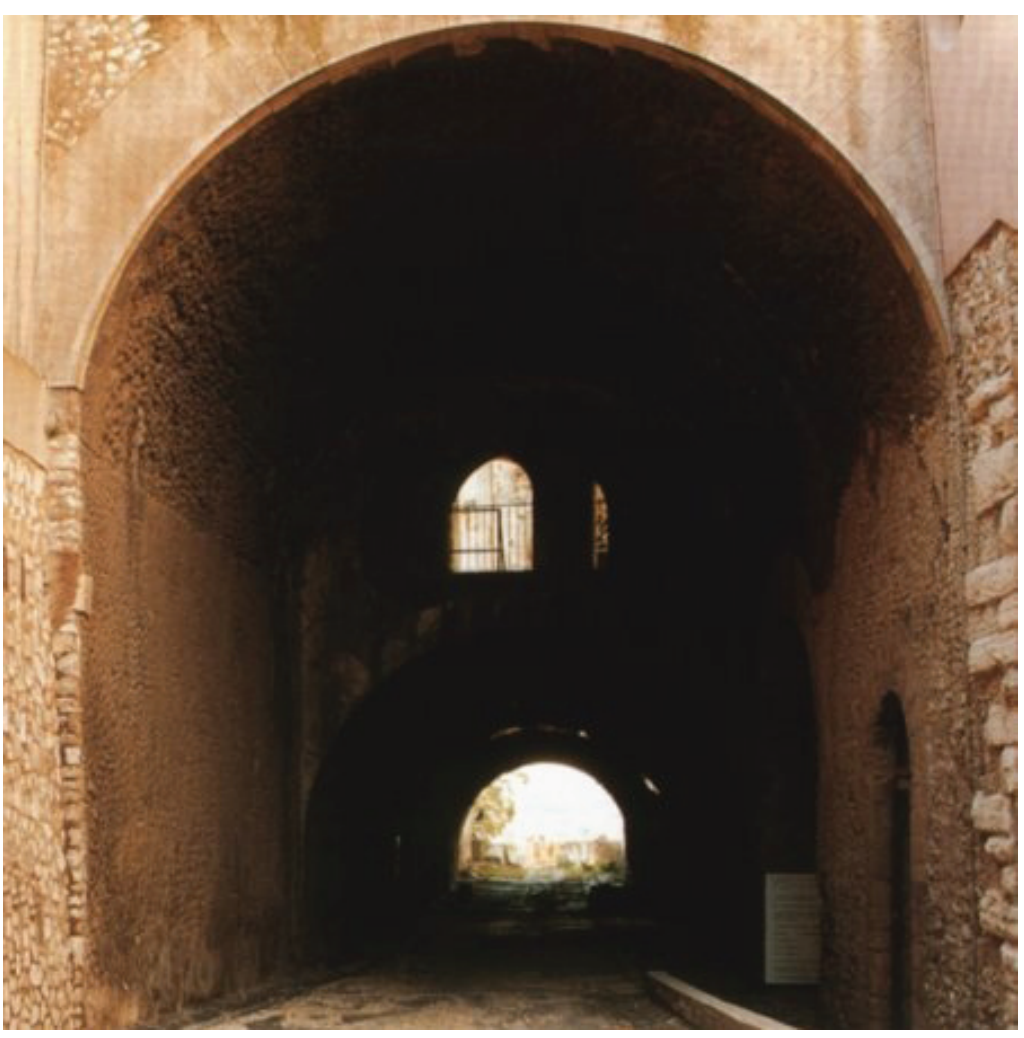

FIG. 2. Via Tecta o Porta oscura, Tívoli ${ }^{25}$

${ }^{25}$ Tomada en junio 2009 de http://www.tibursuperbum.it/ita/monumenti/tempioercole/VicendeStoricheSantuario.htm 


\section{VILLA DE MECENAS}

Textos italiano y español 


\section{Tilla di cllecenate.}

Soi chiamiamo afoluramente Villa di checenate-. questa di cui entriamo a diseorrere, perché quantun. que non bi trovi paloo de antico Jerittore, né lapida, o monumento di vorte alcuna, che cio decisivamen. te dimostri, é nondimeno cerro, ch' egli si dilettava del soggiarno di Sivolizonde avervi doveloe una tit. la corrupondente in magnificenza alla sua Casa di Coma, ed alle sue facolod, esper altra parte vie la rradizione ab immemorabili, che quesra via stata La villa di checenare, corne ché cen questo nome vié chiamata sempure dai Siburtini. Se cö non ei una dimostrazione diretra, precisa, rigorosa, é una indurio. ne fondata, ed ha' a favor suo il titolo del polpepo. Ai Ipereritici troge cose apai piá incerte e conto verribili troveranno in questra parte di scienza anriquaria. S'ultimo dei due estremi é cosa di fatto, e non abbisogna di altra prova; Non vi é mernoria negli archivj privari,o oubblici, che aliro nome in al tri tempi siasi giammai daro a quesro luogo. Iutti gli Scrittori delle cose riburtine nelle loro sopere o pubs blicate, o inedire, cosi la chiamano concordemente. Cosié chiamata nei Commentarj di Gio Gotbellino, - a dir meglio di Enea Jilvio fiecolomini, che fü poi Ponrefice Pio II. Questi Commenrarj benché pubbli. cati piis di too. anne dopo la morte defl" Qutore. 


\section{Villa de Mecenas ${ }^{26}$}

Nosotros llamamos decididamente Villa de Mecenas a ésta de la cual vamos a discurrir, porque aun cuando no se encuentre pasaje alguno de antiguo escritor, ni lápida o monumento de ningún tipo que lo demuestre decisivamente, es cierto, sin embargo, que él se deleitaba en su residencia de Tívoli, donde habría tenido una villa correspondiente en magnificencia a su casa de Roma y a su riqueza; y, por otra parte, existe la tradición ab immemorabili, que ésta haya sido la villa de Mecenas, puesto que con este nombre ha sido llamada siempre por los tiburtinos. Si esto no es una demostración directa, precisa y rigurosa, es una inducción fundada y tiene a su favor el título de propiedad. Los hipercríticos encontrarán en esta parte de la ciencia anticuaria demasiadas cosas muy inciertas y controvertibles. El último de los dos argumentos es un hecho y no requiere de otra prueba. No hay memoria en los archivos privados, o públicos, de que se le haya dado nunca otro nombre a este lugar en otros tiempos. Todos los escritores de los asuntos tiburtinos en sus obras, ya publicadas o inéditas, así la llaman unánimemente. De este modo es llamada en los Comentarios de Giovanni Gobellino ${ }^{27}$ o,

\footnotetext{
${ }^{26}$ Agradezco al Mtro. Luis O. Solís Lozano, director del Archivo Histórico de la Provincia de México, las facilidades otorgadas para la reproducción del manuscrito original; al Lic. Andrés Pérez García, jefe del AHPM, las facilidades otorgadas en la consulta de los materiales del P. Márquez. Finalmente, mi agradecimiento a la Dra. Pía Zanardi Lamberti por haber aceptado revisar la presente traducción.

${ }^{27} \mathrm{Pii}$ secundi pontificis max. Commentarii rerum memorabilium quae temporibus suis contigerunt / à R. D. Ioanne Gobellino... Romae: ex typ., Dominici Basa,
} 
sono opera della prima meta del XV. decoloile. si la chiamano Lilio Giraldi, Govanni Zappi, chn tonio del Ré, Francesco Mavaio, e quindi it Kir cher, il Volpi, e tutti gli altri, che o diretamente, o indirettamenre ne hamo parlaro. fialsro datre. mo, che Mecenate cioè frequentafoe il soggiono di Sivoli labbiano dall' Bde 2q. det lib. 3. 2i Ga zio. Il Socra invitandolo a cenare in bua Casa gli dice, che si sotragga a tutta quello che lo tratiene, abbandoni le cure, e i grandi affari, e gliviene ley. qiadramente a dire, che non rimanga sempre in $t_{-}$ voli a godere di quelle amene vedute.

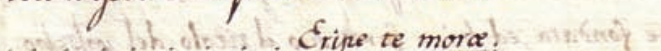
nec semper udum Sibur, er tesule Qedive contempleris arvum, et.

Selegoni juga parricida. cton che voglia mettergli in sospetto il soggiono di Fivoli, perche umido, del quale per alro bap. piamo che Orazio molto se ne compiaceva éjpinn cipalmente per prender morivo di allonramanlo alle grandi occupazioni. Anai pare da questo pafso, che mecenate cola' si wattenepe per distri. gave affari, ed artendere alle cure del governo in compagnia di Augusto. La veduta accennata nei versi di Orazio é appunto quella, che conviene. 
para decir mejor, de Enea Silvio Piccolomini, que fue luego el Pontífice Pío II. ${ }^{28}$ Estos comentarios, si bien publicados más de cien años después de la muerte del autor, (Fol. 2) son obra de la primera mitad del siglo XV. Así la llaman Lilio Giraldi, ${ }^{29}$ Giovanni Zappi, Antonio del Re, Francesco Marzio, y luego Kircher, Volpi y todos los otros, que, directa o indirectamente, nos han hablado de ello. El otro argumento, es decir, de que Mecenas frecuentase su residencia de Tívoli, lo tenemos en la Oda 29 del libro 3 de Horacio. El poeta, invitándolo a cenar en su casa, le dice que se sustraiga de todo aquello que lo retiene, abandone las preocupaciones y los grandes negocios, y él llega a decirle graciosamente que no permanezca siempre en Tívoli para gozar de aquellas amenas vistas.

\section{Eripe te morae; \\ Nec semper udum Tibur, et Aesulae $e^{30}$ \\ Declive contempleris arvum, et Telegoni juga ${ }^{31}$ parricidae. ${ }^{32}$}

No es que quiera insinuarle desconfianza acerca de su permanencia en Tívoli, por su clima húmedo, de lo cual por lo demás sabemos que Horacio se complacía mucho; es principalmente

1584. - [12], 749, [19] p.; $4^{\circ}$. La obra puede ser consultada en la siguiente dirección: http://imagohistoriae.signum.sns.it/TOC_Piccolomini_Commentarii.php

${ }^{28}$ Pío II (Corsignano, 18 de octubre de 1405-Ancona, 14 de agosto de 1464), fue un humanista y religioso italiano, $210^{\circ}$ papa de la Iglesia Católica, desde 1458 hasta su muerte.

${ }^{29}$ Lilio Gregorio Giraldi (Ferrara, 1479-1552). Humanista contemporáneo de Pico della Mirandola. En su obra De Deis Gentium, editada en Basilea en 1548, menciona esporádicamente anotaciones sobre hallazgos arqueológicos y colecciones de antigüedades de Roma, que probablemente haya conocido de manera directa durante su estancia en esta ciudad.

${ }^{30}$ Aefula o Aesula, Éfula, antigua ciudad del Lacio, situada al este de la antigua Gabii o Gabies, y al sudeste de Tíbur, actual Tívoli.

${ }^{31}$ El mito de Telégono, hijo de Odiseo y Circe (para algunos de Calipso) está relacionado con la fundación de Túsculo en los Montes Albanos, al sur de Roma.

32 Sustráete de la demora; / no siempre contemplarás Tívoli húmedo, / y de Éfula un campo en pendiente, / y las cumbres del parricida Telégono. 
- llla posizione della nosira villa, che dne ilpen. dio, e la campagna di Tivoli domina $i$ colli del $\mathcal{S}_{w}$ rido. La localita non pai meglio corris pondere ed il testo addotto, se é l'unico che adduore dicpofa, époi cosi chinro, che enon lascia luogo a dubitare, che Mecenate dpepo, e lungamente in Fiveli si

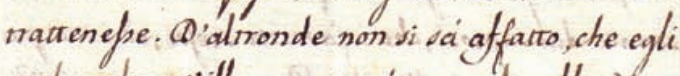
ancepe ntera villa mentre écento, che abbondava in ricchezze, ed ecoedeva nel tujpo. Equatialiro wi era Cavaliere ?omano mediacremente facoltoso, che non avefue la dua villa quando vaguiamo, che generalmente almen due ne bavevano, una al mare per l'inverno, lialira alla montagna per pas. aryi testate? Slinio il giovine benche non fope uomo ricchifoimo, pure olre il suo Laurentine, do. $v e$ andava, come vanno anche a giorni nostri in quelle vicinanze i dignori Zomani nelli inverno, aveva la sua villa in Joscana lille falde degli Appennini. Quesre due furono le principalis non. dimeno dalla enistola 6. del lib. simpariamo, che altre n'eble nel Suscolo, in Pivoli, in Salesirina. tabes causas, cur ego Thuseos meos tusculanis, tifurtinid, proenestinisque imeis proponam. = ques to é un' esempio degli infiniti, che si potrebbers. addurre. Di piu, se mecenate vifabbrico in homa खरी 
para encontrar un pretexto para alejarlo de las grandes ocupaciones. Al contrario, parece por este pasaje que Mecenas se quedaba en aquel lugar para despachar sus negocios y atender los asuntos del gobierno en compañía de Augusto. La vista mencionada en los versos de Horacio es precisamente aquella que concuerda (Fol. 3) con la posición de nuestra Villa, que, más allá de la pendiente y la campiña de Tívoli, domina las colinas de Túsculo. La localidad no puede corresponder mejor, y el texto citado, si bien es el único que se puede aducir, es tan claro, que no deja lugar a duda que Mecenas a menudo y por mucho tiempo se quedase en Tívoli. Por otra parte, no se sabe en absoluto que él tuviese otra Villa, mientras se sabe con certeza que sus riquezas eran abundantes y sus lujos extraordinarios. ¿Había acaso algún caballero romano mediocremente acaudalado que no tuviese una villa, cuando sabemos que generalmente al menos tenían dos, una en la playa para el invierno, otra en el monte para pasar el verano? Plinio el joven, a pesar de que no era un hombre riquísimo, sin embargo, además de su Villa Laurentina, a donde se dirigía - como van también en nuestros días a aquellas zonas los señores romanos en el invierno - tenía su villa en la Toscana, a las faldas de los Apeninos. Estas dos villas eran las principales; con todo eso, por la epístola 6 del lib. 5 sabemos que tenía otras en Túsculo, en Tívoli, en Palestrina: Habes causas, cur ergo Thuscos meos tusculanis, tiburtinis, praenestinisque meis praeponam. ${ }^{33}$ Esto es un ejemplo de los muchos que se podrían aducir. Más aún, si Mecenas se construyó en Roma (Fol.

\footnotetext{
${ }^{33}$ Hor., Ep. V, 6. Tienes las razones de por qué antepongo mis (propiedades) toscanas a las tusculanas, tiburtinas y prenestinas.
} 
La casa, égli orri pé quali a nuin alira cedevaned la mragrificenza, era troppo naturale, che si fabbri. vape una villa ugualmente magnifica, e grandio. a. Capio, Quintitio Varo e molrifoimi altri $3 \dot{e}$ primi Signori di Boma avevano in Sivoli la villa. Cre vee poi la presungione a favore della villa di me. cenate dal sapersi, che i faworiti stepo, ed i vusi püu cari amici Orazio, Virgilio, Properzio avevano in $3 i$. voli, cioé in quelle vicinan ze la loro, forse prer epe. re cosi piti vicini, edirei quasi per far corte allo. ro bencfattore. firro Ligorio, ed altri thanno chia mara ancora villa di augusto. quesra denomina zione anziché distruggere ta nosna, verve ad aw. valorarla, ed avere appartenuro ad augusto é un. altro argomento che dá forza ai già addotri per provarla villa di Mecenate. Oione nel lib. S5. ci dice, che Augusto fü-lasciato erede da mecenate, dunque questa villa ancora pajo in sua proprieta. Saypiamo in mille maniere, che Augusto frequen. tava la casa di mecenate, onde si potrebbe da ques to solo inferire, che augusto verifoe spepo a trat. tenervisi; ma da un pajoo di Svetonio non che lo popiamo, ma quasi lo dobbiamo inferire. $=$ Ex decefoibus pracipue frequentavit marivima, insulas. que Campania, aut proxima Urbi onpida, anuvium,

Sira. 
4) la casa y los jardines, por los que no era segundo a nadie en la magnificencia, era muy natural que se edificase una villa igualmente magnífica y grandiosa. Casio, Quintilio Varo, y muchísimos otros de los primeros señores de Roma tenían en Tívoli una villa. Se acrecienta por tanto la hipótesis en favor de la villa de Mecenas al saber que sus mismos protegidos y sus más queridos amigos Horacio, Virgilio y Propercio tenían en Tívoli, esto es, en aquellas cercanías, sus villas, quizá por estar así más próximos, y diría que casi para acompañar a su benefactor. Pirro Ligorio y otros la han llamado también Villa de Augusto. Esta denominación más que destruir la nuestra, sirve para apoyarla; y haber pertenecido a Augusto es otro argumento que da fuerza a los ya aducidos para demostrar que la villa era de Mecenas. Dión en el lib. $55^{34}$ nos dice que Augusto quedó como heredero de Mecenas, de ahí que esta villa pasó también a su propiedad. Sabemos de mil maneras que Augusto frecuentaba la casa de Mecenas, hecho por el que se podría sólo inferir que Augusto venía con frecuencia a hospedarse; sin embargo, por un pasaje de Suetonio, no sólo podemos, sino que casi debemos colegirlo. Ex secessibus praecipue frequentavit maritima, insulasque Campaniae, aut proxima Urbi oppida, Lanuvium, (Fol. 5) Praeneste, Tibur, ubi

\footnotetext{
${ }^{34}$ Dion $55,7$.
} 
Praneste, Jibur ubi eriam in porricibus templi tier. culis perscape juo dixir se de frequenró Tivoli in ma nierarche spelpo vemiva a giadicarre indile Cause. nel tempio di Ercole, chivi dice, che non ne prendesse motivo a cio fare dall elsere ivi la villa del suo. amico, che serviva quasi di vichiamo? Santo puiu, che dallo stepo. Storico saypiamo, che fece uso dei bagni dell'acque albule, per it quale uso era molto a portata questa villa $=$ at quories nervorum causa marinis, albulisque calidis utendum eper. de. Di priü se ta magnificenza della villa non era contorme alla maniera di pensare di Auguto, it che $=$ ampla, er operosa pratoria gravabarur $=q u e s$ to fa' vedere, che non era stato egli il fondatore, ed il primo padrone; vi erano pero delle cose parte na. turali parte artefacte, ch'efoo gradiva nei luoghi di sua abitazione $=$ sua vero quamvis modica non tam statuarum, tabularumque picrarum ornatu. quam xistis, et nemoribus excoluir - Equesta fab brica appunto in gran parte consisteva in xisti, come diciam noi in porrici, e loggie, $i$ quali mentre godevano la piu amerra veduta, dourastavano al dirupo del elivo tiburrino tulto ricoperto , com'é anche adepo, di boscaglie: Se vtanze intermedie. le quali non prendono lime, che vallo ponte ditua 
etiam in porticibus templi Herculis persaepe jus dixit. ${ }^{35} \mathrm{Si}$ frecuentó Tívoli de manera que a menudo iba a juzgar allí las causas en el templo de Hércules, ¿quién nos dice que un buen motivo para hacerlo no fuera el hecho de que allí estaba la villa de su amigo, que servía casi de incentivo? Tanto más, que por el mismo historiador sabemos que hizo uso de las Aguas Álbulas, ${ }^{36}$ para cuyo propósito estaba muy al alcance esta villa. At quoties nervorum causa marinis, albulisque calidis utendum esset, ${ }^{37}$ etc. Más todavía, si la magnificencia de la villa no era conforme a la manera de pensar de Augusto, que ampla, et operosa praetoria gravabatur, ${ }^{38}$ esto hace ver que él no había sido el fundador y primer dueño; había empero cosas en parte naturales, en parte artificiales, que él apetecía en los lugares de su habitación: sua vero quamvis modica non tam statuarum, tabularumque pictarum ornatu, quam xistis, et nemoribus excoluit. ${ }^{39} \mathrm{Y}$ esta construcción precisamente consistía en gran parte en xisti, o como decimos nosotros, en pórticos y en terrazas cubiertas, los cuales, mientras gozaban la vista más amena, dominaban el peñón de la pendiente tiburtina toda recubierta, como lo está también ahora, de matorrales. Las estancias intermedias, las cuales reciben luz sólo de las

\footnotetext{
${ }^{35}$ Suet., Aug. 72, 2. En sus retiros frecuentó en particular las costas y las islas de Campania o ciudades próximas a Roma: Lanuvium, Preneste, Tíbur, donde también, asiduamente bajo los pórticos del templo de Hércules, impartió justicia.

${ }^{36}$ Fuentes de aguas sulfurosas en las proximidades de Tíbur, llamadas Acque Albule o Bagni di Tivoli.

${ }^{37}$ Idem 82, 2. Pero cuántas veces a causa de los nervios debía valerse de los baños marinos y cálidos de Álbula.

${ }^{38}$ Idem 72, 3. No gustaba de las casas de campo grandes y costosas.

${ }^{39}$ Ibidem. En sus (villas), aunque modestas, cuidó no tanto de las estatuas y de los cuadros de ornato como de los pórticos y bosques.
} 
te situate nei porrici adiacenti, dovevano epere liabirazione piu difesa, e riparata dal caldo dell. estare, e questo stejo pare molvo confacente ai desi. derj,ed alla mollezza di mecienate. Forse niente meno ad Auquito poreva gradire quella maniena divabitare, il quale sappiamo, che molto vicercava le comadita, ed evitava tuto cio, che potepe necargli fas. tidio. liangiava in leatiga di noure, ed a piccole gion nate. = Irimera lectica, er noctibus fere, eagno lenta, ac minuta faciebut, ut Pranevte, vel gibun-biduo. procederes:- Ma parrioslarmente in quella specie di camere pare, che gradifue di abitared dape in pe ristylio, valiente aqua, arque eriam ventilante ali quo cubabat: Non puó meglio convenire, che alla fabbrica della quale partiamo. Ti era il peristitio. eviera l'acqua, che remoreggiava. Sutto ció éum mucchio di piccole congetzure é vero, ma unite servono a corroborario f'un l'altra, e tutte insieme sosrengono la nosrra tesi. On peso anche piú grande le dá il porrieo lungo 400 pafoi, cheresisteva secondo una iserizione, il quale univa la villa ai porrici del tempio di Ercole. adefor bavi averlo indiearo, pen. ché par fauto a posta acciocché Auguvio enorapeal coperto dalla silla nnei porrici del tempio sudeuto. 2? illaxione é molto naturale, e con rutti gli altividaris 
puertas colocadas (Fol. 6) en los pórticos adyacentes, debían ser las habitaciones más resguardadas y protegidas del calor del estío; y esto mismo parece muy conforme a los deseos y a la delicadeza de Mecenas. Quizá a ningún otro más que a Augusto podía agradar aquella manera de vivir, de quien sabemos que buscaba mucho las comodidades y evitaba todo lo que pudiese ocasionarle fastidio. Viajaba en litera, de noche y en breves jornadas: Itinera lectica, et noctibus fere, eaque lenta, ac minuta faciebat, ut Praeneste, vel Tibur biduo procederet. ${ }^{40}$ Mas parece que gustaba de habitar particularmente en esta especie de recámaras: saepe in peristylio, saliente aqua, atque etiam ventilante aliquo cubabat. ${ }^{41}$ No puede corresponder mejor que a la construcción de la cual hablamos. Tenía peristilo y había agua que rumoreaba. Todo esto es un montón de pequeñas conjeturas, es verdad, pero unidas sirven para que se corrobore una con otra, y todas juntas sostengan nuestra tesis. Un peso también muy grande le da el pórtico de 400 pasos de largo, que existía siguiendo una inscripción, el cual unía la villa a los pórticos del templo de Hércules. Ahora sea suficiente haberlo indicado, porque parece hecho expresamente para que Augusto entrase, a cubierto, desde la villa en los pórticos de dicho templo. La ilación es muy natural y con

\footnotetext{
${ }^{40}$ Idem 82, 1. Realizaba sus viajes en litera, casi siempre de noche, poco a poco y en cortos trayectos, de suerte que en dos días llegaba a Palestrina o Tívoli.

${ }^{41}$ Ibidem. Con frecuencia dormía en el peristilo, cerca de una fuente e incluso abanicado por alguien.
} 


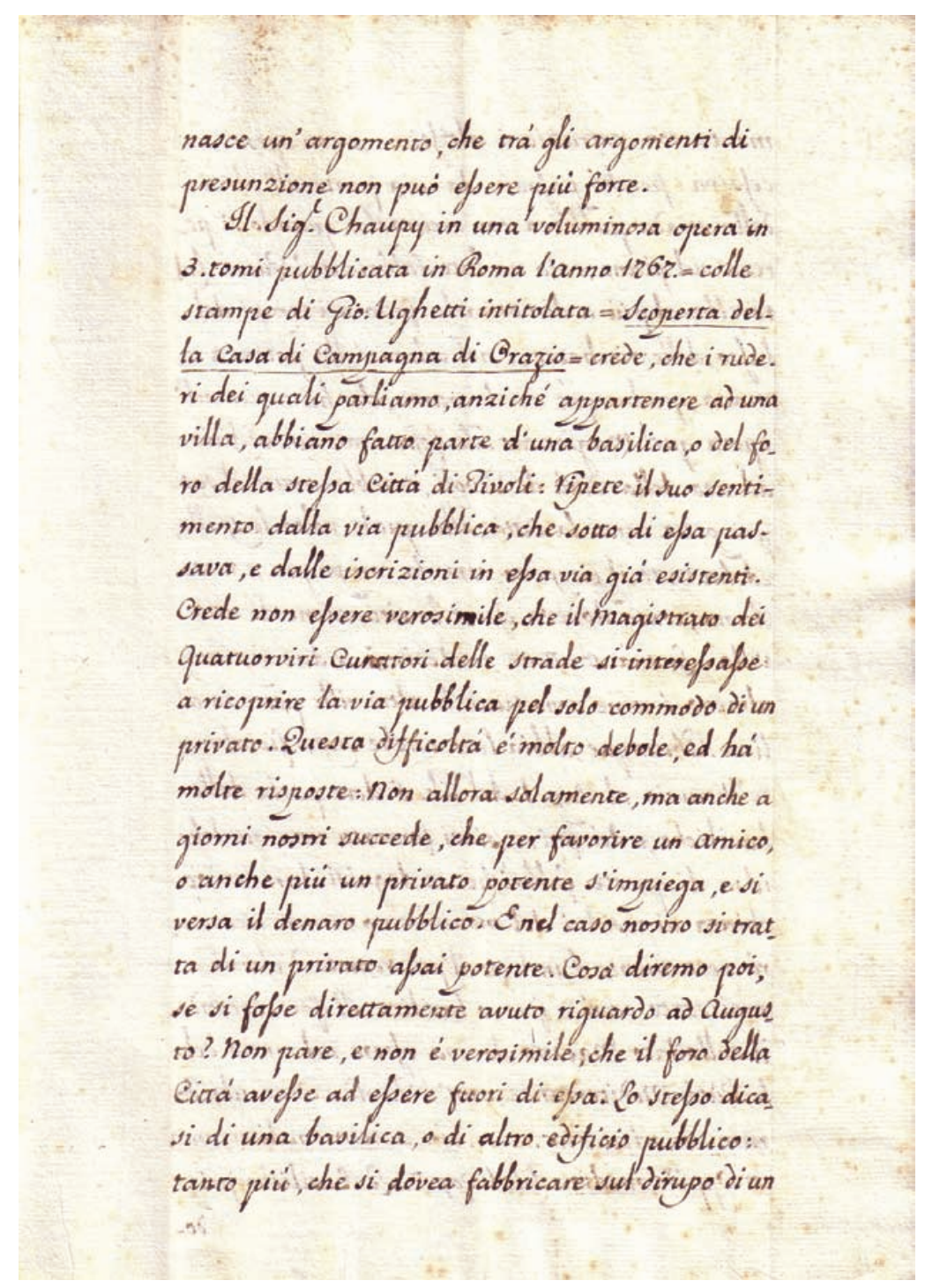


todos los otros datos (Fol. 7) nace un argumento que, entre los argumentos de suposición, no puede ser más fuerte.

El señor Chaupy en una voluminosa obra en 3 tomos, publicada en Roma en el año de 1767 en la imprenta de Giorgio Ughetti, intitulada Scoperta della Casa di Campagna di Orazio, cree que las ruinas de las que hablamos, en lugar de pertenecer a una villa, hayan formado parte de una basílica o del foro de la misma ciudad de Tívoli: refuerza su parecer a raíz de la vía pública que pasaba debajo de ésta, y a partir de las inscripciones ahí gravadas. Cree que no es verosímil que el magistrado de los cuadrúnviros ${ }^{42}$ encargado de las calles se interesase en recubrir la vía pública para comodidad de un solo particular. Esta dificultad es muy débil, y tiene muchas respuestas: no sólo en aquel entonces, sino en nuestros días sucede que, para favorecer a un amigo o, más aún, a un particular poderoso, se emplea y se gasta el dinero público. Y en el caso nuestro se trata de un particular muy poderoso. ¿Qué diríamos además, si se hubiese directamente tenido deferencia hacia Augusto? No parece y no es verosímil que el foro de la ciudad hubiese de estar fuera de ella. Lo mismo dígase de una basílica o de otro edificio público, tanto más, que debía construirse sobre el acantilado de un (Fol. 8) monte con construcciones altísimas

\footnotetext{
${ }^{42}$ Se refiere a los cuatro magistrados que en Roma tenían a su cargo el cuidado y conservación de la pavimentación y restauración de las calles de la ciudad.
} 


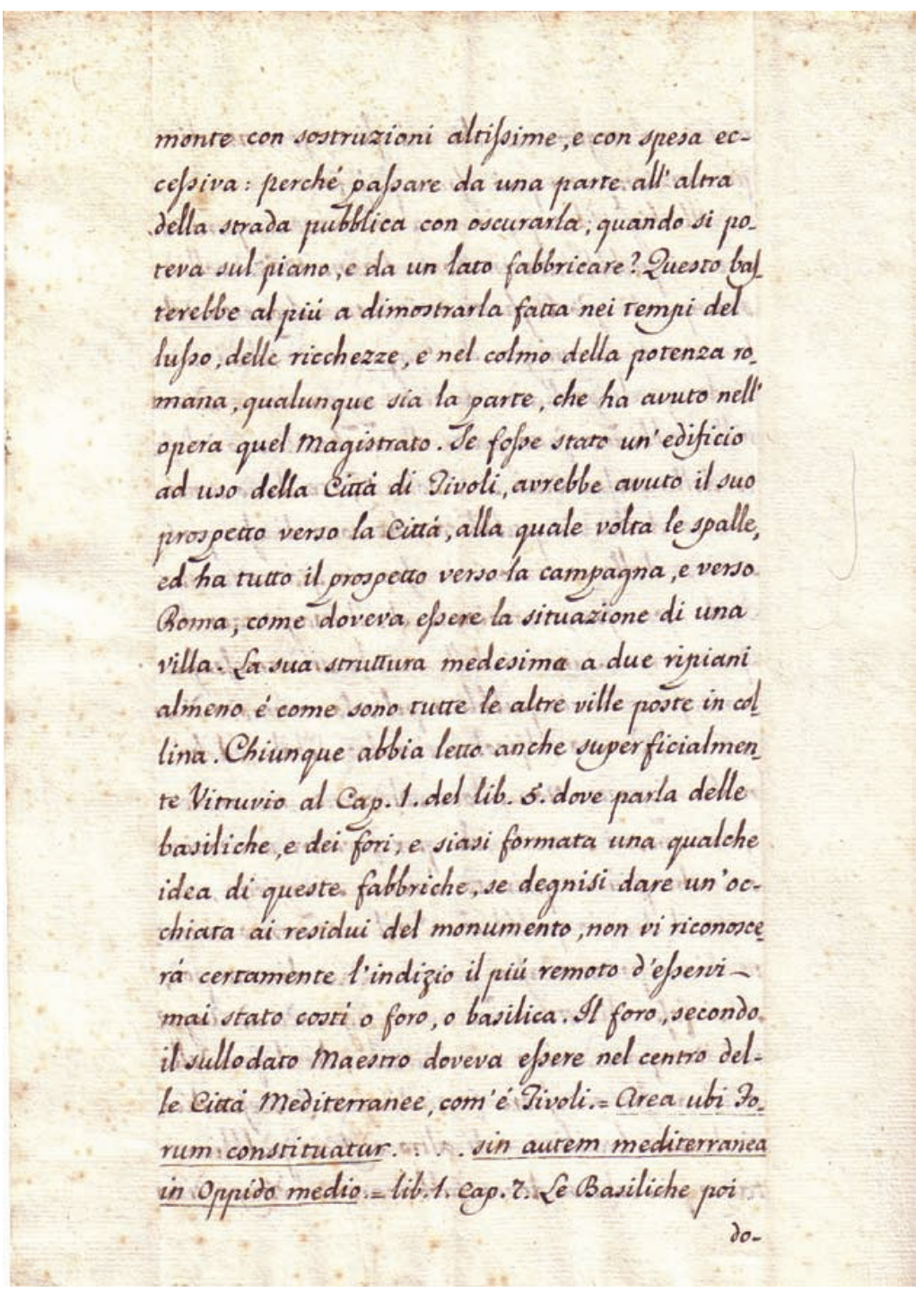


y con gasto excesivo: ¿por qué pasar de un lado al otro de la calle pública a cubierto, ${ }^{43}$ cuando se podía construir sobre lo llano y de un solo lado? Esto bastaría a lo más para demostrar que fue hecha en tiempos de lujo, de riqueza y en el apogeo de la potencia romana, cualquiera que sea la parte que tuvo en la obra aquel magistrado. Si hubiese sido un edificio para uso de la ciudad de Tívoli, habría tenido su frente hacia la ciudad, a la cual da la espalda, y tiene el frente hacia la campiña y hacia Roma, como debía ser la ubicación de una villa. Su estructura misma con dos niveles al menos es como son todas las otras villas ubicadas en colina. Cualquiera que haya leído aun superficialmente a Vitruvio en el cap. 1 del lib. 5. donde habla de las basílicas y de los foros y se haya formado alguna idea de estas construcciones, si se dignara dar una ojeada a los restos del monumento, no reconocerá ciertamente el indicio más remoto de que haya estado nunca allí algún foro o basílica. El foro, según el referido maestro, debía estar en el centro de las ciudades mediterráneas, como es Tívoli: Area ubi Forum constituatur... sin autem mediterranea in Oppido medio. ${ }^{44}$ lib. 1 cap. 7. Las basílicas, por otra parte, de- (Fol. 9) bían estar

\footnotetext{
${ }^{43}$ Se refiere, al parecer, a la Via Tecta o Porta oscura (cf. fig. 2).

${ }^{44}$ Vitr. I, 7, 1. El área donde se construirá el foro... si fuera una ciudad mediterránea, será en medio de la ciudad.
} 


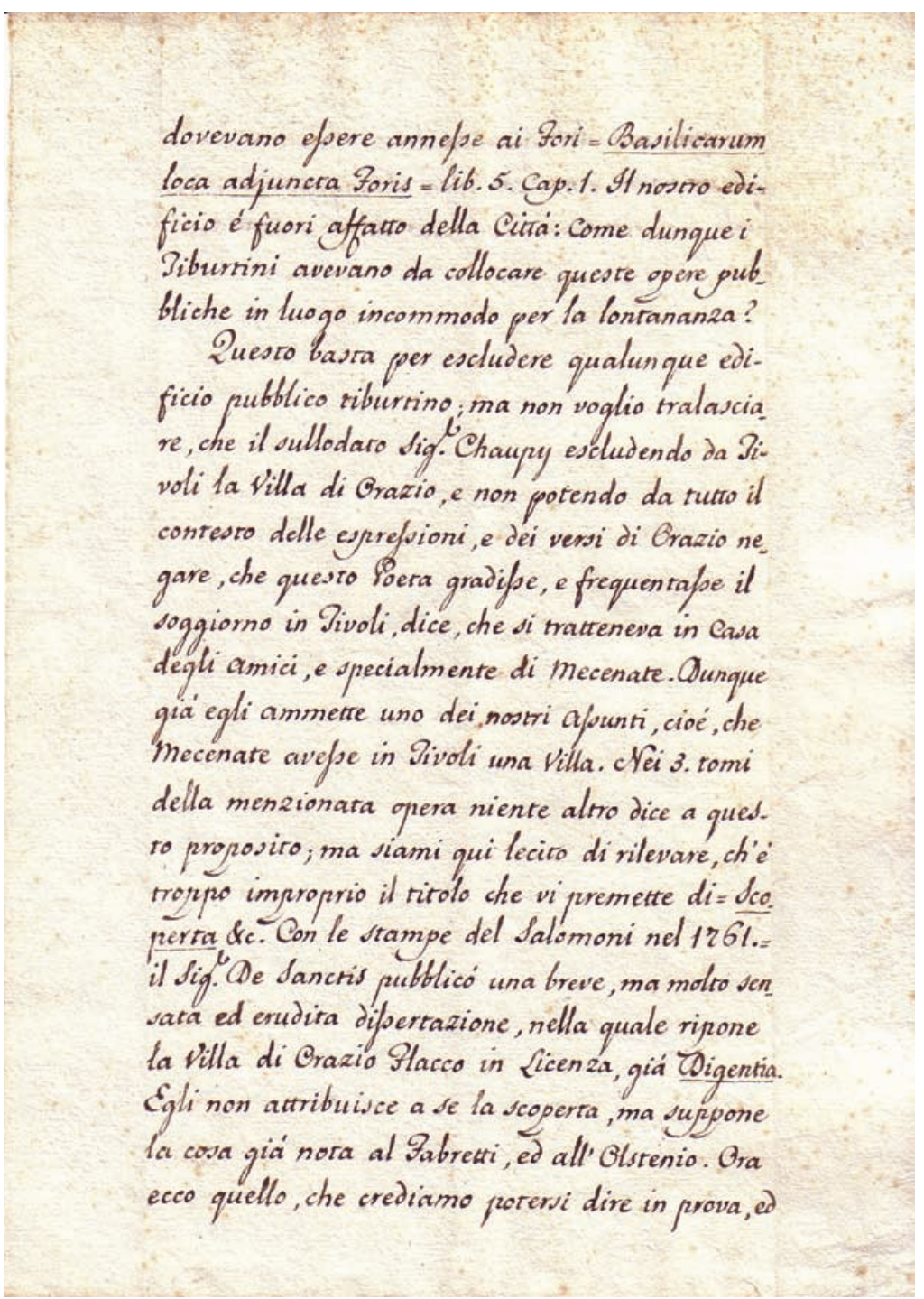


anejas a los foros: Basilicarum loca adjuncta Foris ${ }^{45}$ lib. 5. cap. 1. Nuestro edificio está por completo fuera de la ciudad. ¿Cómo, entonces, los tiburtinos habrían de colocar estas obras públicas en un lugar incómodo por la lejanía?

Esto basta para excluir algún edificio público tiburtino; mas no quiero pasar por alto al mencionado señor Chaupy, que, aunque niegue la presencia en Tívoli de la villa de Horacio, y no pudiendo negar por todo el contexto de las expresiones y de los versos de Horacio que este poeta gustara y frecuentara la estancia en Tívoli, dice que se hospedaba en casa de los amigos y especialmente de Mecenas. Así pues, ya él admite uno de nuestros argumentos, es decir, que Mecenas tuviera en Tívoli una villa. En los tres tomos de la obra citada, ninguna otra cosa se refiere a este propósito, mas séame aquí lícito advertir que es demasiado impropio el título que ahí pone de Scoperta, etc. ... En la imprenta del Salomoni en 1761, el señor De Sanctis publicó una breve, pero muy sensata y erudita disertación, en la cual coloca la villa de Horacio Flaco en Licenza, antes Digentia. ${ }^{46}$ Él no se atribuye a sí mismo el descubrimiento, mas supone el asunto ya conocido por Fabretti y por Holstenio. ${ }^{47}$ Ahora he aquí aquello que creemos

\footnotetext{
${ }^{45}$ Idem V, 1, 4. Los lugares de las basílicas (conviene se construyan) junto a los foros.

${ }^{46}$ Arroyo o pequeño río en el país de los Sabinos (hoy Licenza).

${ }^{47}$ Lucas Holstenius (1596-1661). Humanista, geógrafo e historiador alemán convertido al catolicismo.
} 


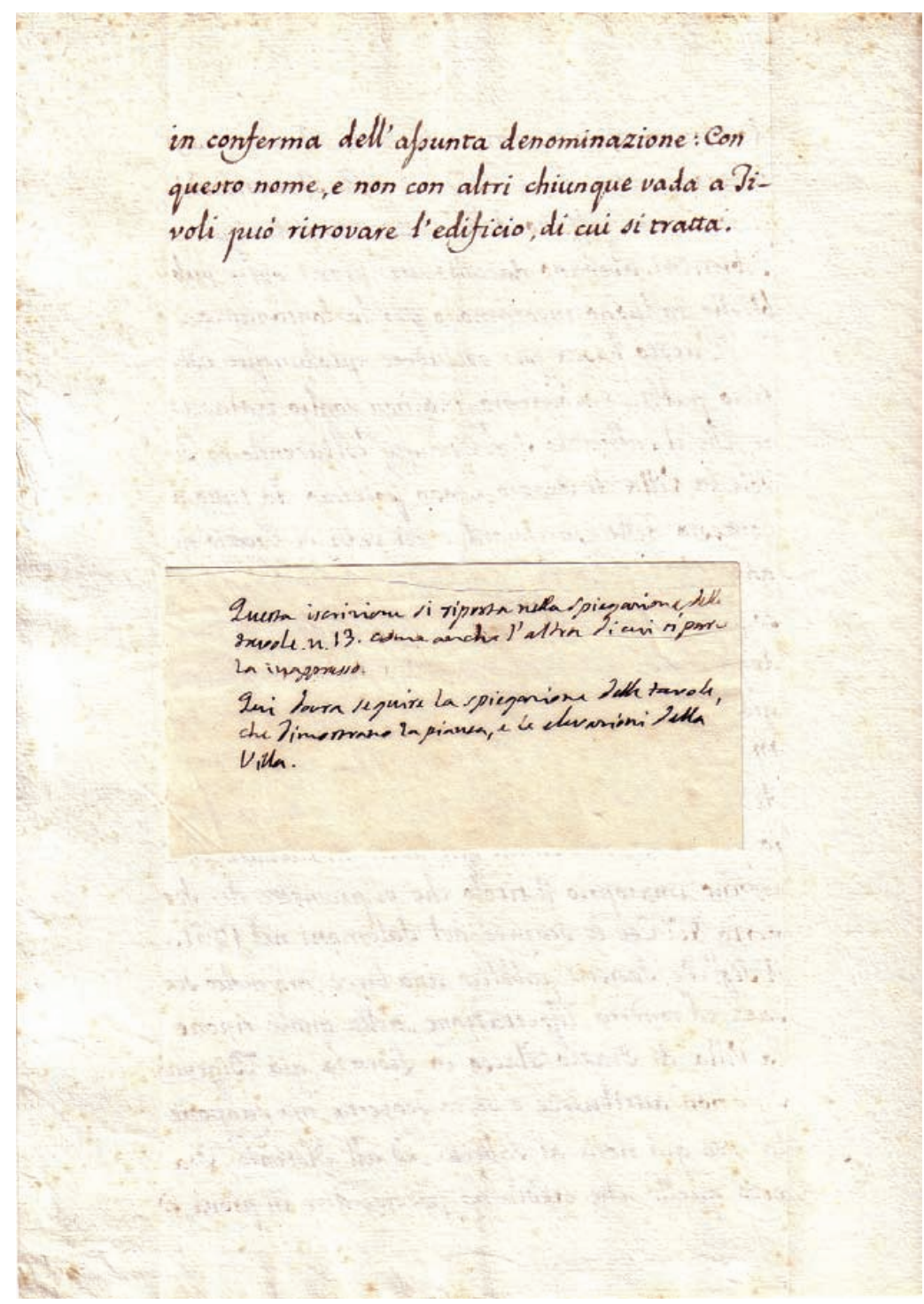


se puede decir como prueba y (Fol. 10) como confirmación de la dicha denominación: con este nombre y no con otro quien sea que vaya a Tívoli puede encontrar el edificio del cual aquí se trata. 


\section{BIBLIOGRAFÍA}

Castellanos de Losada, Basilio, Álbum de Azara: Corona científica, literaria, artística y política que las Universidades, Academias, Maestranzas... consagran a la ... memoria del célebre diplomático y... literato español... D. José Nicolás de Azara y Perera... Madrid, Alejandro Fuentepedro, 1856.

-, Biografía del célebre diplomático José Nicolás de Azara, Madrid, D. G. González, 1850.

CIPRIANI, Giovanni Battista, Itinerario figurato degli edifizi più rimarchevoli di Roma, Roma, s. n., 1835.

Couto, Bernardo, "Márquez, Pedro José", en Diccionario Universal de Historia y de Geografía, T. V, México, Impr. de F. Escalante, 1854, pp. 143-144.

DeCORME, Gerardo, Varones ilustres de la provincia de México muertos en el siglo diez y nueve. Compilación histórica de varios autores (texto mecanografiado, Biblioteca Dr. Eusebio Dávalos Hurtado del MNAH-INAH, Fondo jesuita, carpeta XXV, doc. 4/19: "Pedro José Márquez").

Fernández Agudo, M Ma Pilar, y M $\mathrm{M}^{\mathrm{a}}$ Ángeles SÁnchez, "Índice de cargos académicos de la Real Académica de Bellas Artes de San Fernando en el siglo XVIII", Academia, 67, 1988, pp. 371-458.

García Melero, José Enrique, Arte español de la Ilustración y el siglo XIX, en torno a la imagen del pasado, Madrid, Encuentro, 1998.

GutiérReZ HaCes, Juana, "La preparación de un diccionario. Los apuntamientos... pertenecientes a la arquitectura del padre Pedro José Márquez", Anales del IIE, 16, 65, 1994, pp. 85-88.

Márquez Durán, Pedro José, Delle case di città degli antichi Romani, secondo la dottrina di Vitruvio. Esposta da D. Pietro Márquez, Messicano, Roma, Presso il Salomoni, 1795.

_, Dell'ordine dórico ricerche dedicate alla Reale Academia di S. Luigi di Zaragoza..., Roma, Presso il Salomoni, 1803.

-, Delle ville di Plinio il giovane, con un appendice sugli atri della $S$. Scrittura, e gli scamilli impari di Vitruvio, Roma, Presso il Salomoni, 1796.

-, Esercitazioni architettoniche sopra gli spettacoli degli antichi, con appendice sul bello in generale, Roma, Presso il Salomoni, 1808.

-, Illustrazioni della villa di Mecenate in Tivoli, Roma, Stamperia de Romanis, 1812.

MÉNDEZ PlanCARTe, Gabriel (comp.), Humanistas del siglo XVIII, México, UNAM (Biblioteca del Estudiante Universitario, 24), 19914. 
Mora Rodríguez, Gloria, y Beatrice CACCIOTTI, "Coleccionismo de antigüedades del clasicismo: relaciones entre Italia y España en el siglo XVIII", Hispania: Revista española de historia, v. 56, núm. 192, 1996.

NiBby, Antonio, Viaggio Anticuario ne' contorni di Roma, Roma, Presso Vincenzo Poggioli, 1819.

Orozco Muñoz, Julio, Pedro José Márquez, su vida y su obra, 1741 1941, México, Historia y Poesía, 1941.

SÁnchez Espinosa, Gabriel, La biblioteca de José Nicolás de Azara, Madrid, Calcografía Nacional, Real Academia de Bellas Artes de San Fernando, 1997.

-, Las memorias de José Nicolás de Azara (Ms. 20121 de la BNM), Frankfurt am Main, Lang, 1993.

VALDÉS GARCÍA, Hilda J., "De los baños romanos al temazcalli prehispánico: la interpretación de Vitruvio V, 10 por Pedro José Márquez (1741 - 1820), Nova tellus, 26.2, 2008, pp. 251-270.

-, Obra de romanos. La formación del arquitecto, métodos de construcción y materiales según Vitruvio, Tesis de Maestría, FFyL, UNAM, 2007.

-, "Rifflezioni sopra $i$ muri di pietre irregolari. Discurso inédito del jesuita mexicano Pedro José Márquez (1741-1820)", ponencia presentada en el XXII Encuentro de Investigadores del Pensamiento Novohispano, llevado a cabo en la Universidad de Guanajuato, México, del 4 al 6 de noviembre de 2009.

-, "Sopra le antiche strutture, un discurso inédito de Pedro José Márquez", ponencia presentada en el Coloquio Internacional "El clasicismo en la época de Pedro José Márquez (1741-1820). Arqueología, filología, historia y teoría arquitectónica", llevado a cabo en la Real Academia de San Fernando, Madrid, España. Del 27 al 31 de octubre de 2009.

\section{Sites}

http://www.tibursuperbum.it/ita/monumenti/tempioercole/VicendeStoricheSantuario.htm Consultado en junio de 2009. 